This is the post peer-review accepted manuscript of:

F. Tonini, C. Raffaelli, B. M. Khorsandi, S. Bjornstad and R. Veisllari, "Converged Fronthaul/Backhaul based on Integrated Hybrid Optical Networks," 2018 Asia Communications and Photonics Conference (ACP), Hangzhou, 2018, pp. 1-3.

The published version is available online at:

https://doi.org/10.1109/ACP.2018.8595915

(C) 2018 IEEE. Personal use of this material is permitted. Permission from IEEE must be obtained for all other uses, in any current or future media, including reprinting/republishing this material for advertising or promotional purposes, creating new collective works, for resale or redistribution to servers or lists, or reuse of any copyrighted component of this work in other works. 


\section{Converged Fronthaul/Backhaul based on Integrated Hybrid Optical Networks}

\author{
Federico Tonini \\ $D E I$ \\ University of Bologna \\ Bologna, Italy \\ f.tonini@unibo.it
Raimena Veisllari
Transpacket \\ $A S$ \\ Oslo, Norway \\ raimena.veisllari@transpacket.com
}

\author{
Bahare M. Khorsandi \\ DEI \\ University of Bologna \\ Bologna, Italy \\ bahare.masood@unibo.it
}

University of Bologna

Bologna, Italy

carla.raffaelli@unibo.it

\author{
Steinar Bjornstad \\ Transpacket \\ $A S$ \\ Oslo, Norway \\ steinar.bjornstad@transpacket.com
}

\begin{abstract}
Integrated hybrid optical network is investigated to carry both fronthaul and backhaul traffic on an Ethernet optical channel. Evaluation of the additional backhaul throughput is presented to demonstrate the effectiveness of the approach.

Index Terms-hybrid optical networks, fronthaul, backhaul, C-RAN, convergence.
\end{abstract}

\section{INTRODUCTION}

Centralized/Cloud Radio Access Network (C-RAN) is a promising technique enhancing mobile network performance and flexibility while reducing the network cost [1]. Common Public Radio Interface (CPRI) [2] is nowadays the most common protocol to transport data in the fronthaul $(\mathrm{FH})$ segment of C-RAN between radio sites and baseband hotels/mobile edge computing. Being a TDM-based protocol, it poses strict capacity, latency and latency variation requirements on the transport network. More recent packet-based functional splitting protocols, such as eCPRI [2], have been released, while splits proposed by 3GPP [3] are on their way of standardization. However, so far, the majority of the proposed splits concern the radio physical layer and do not relax the strict delay requirements on the FH transport.

In recent proposals [1], the highly demanding traffic generated by these functional splits is transported together with traditional backhaul (BH) traffic over a common optical infrastructure by sharing the same fiber links using different wavelengths. WDM solutions solve the latency issues but may limit system scalability and lead to low resource utilization in the presence of many antennas. This is due to the provisioning of one wavelength channel per antenna/sector. Hence, a technique for improving the optical channel utilization while meeting the strict performance requirements is investigated in this paper.

A packet-based integrated hybrid optical network (IHON) with ultra-low packet delay variation (PDV) services and statistically multiplexed traffic, is proposed in [4]. Implementation based on Ethernet has been demonstrated in C-

This work is partially funded by EU H2020 5G-PICTURE project.
RAN [8], assuming CPRI traffic transparently transported by a wavelength and using the leftover capacity for BH traffic. However, to prevent the FH traffic from PDV caused by BH traffic insertion, IHON adds a fixed delay, corresponding to the maximum duration of a $\mathrm{BH}$ frame, to all $\mathrm{FH}$ packets.

In this work the IHON architecture is extended with preemption, allowing the additional delay on the FH traffic to be lowered and independent of the maximum $\mathrm{BH}$ frame size. IEEE Time Sensitive Networking (TSN) group has proposed a FH standard, the IEEE 802.1CM [9], which assumes high priority $\mathrm{FH}$ frames to preempt lower priority $\mathrm{BH}$ frames, to introduce low delay. However, due to the time needed by the preemption process, the PDV is not minimized. Preemption in IHON, instead, allows lowered delay and zero PDV. Results obtained by simulation demonstrates that the approach reaches a remarkable throughput of the $\mathrm{BH}$ traffic on the shared Ethernet optical channel and hence, a good exploitation of the leftover FH capacity.

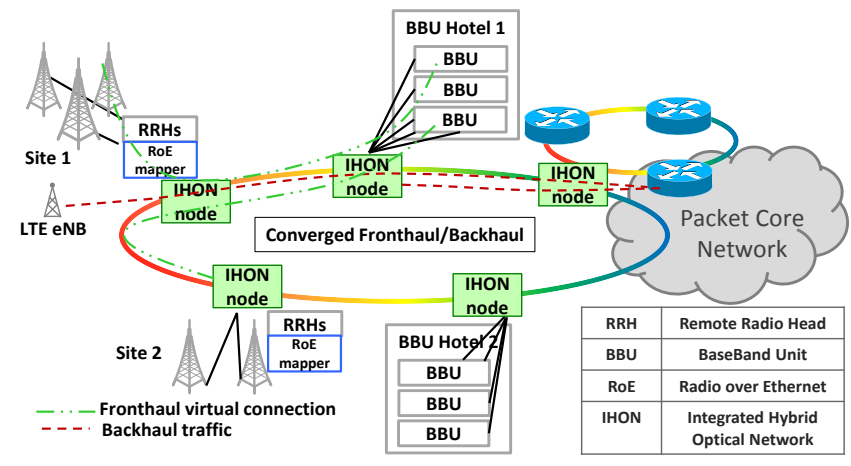

Fig. 1. Converged fronthaul/backhaul scenario.

\section{INTEGRATED HYBRID OPTICAL NETWORK IN C-RAN}

Fig. 1 shows a sample ring topology implementing a CRAN. The baseband unit (BBU) serving a remote radio head 
(RRH) can be activated in different hotels for resource optimization, service continuity or energy efficiency, thus requiring dynamic association between RRHs and BBUs. Moreover, traditional base stations (e.g., LTE eNB) may also be present in the same area, requiring connection to the core network. As a consequence, both FH and $\mathrm{BH}$ traffic need to be transferred on each network segment.

IHON was first proposed in [4], analyzed in [5] and experimentally proved in [6]. It consists of a packet-based technique which can be implemented in an Ethernet switch, enhanced with guaranteed time transparent service. A fixed delay $(\Delta)$ is added to guaranteed traffic so that statistically multiplexed traffic, transmitted on the same optical channel, is allowed to be preempted by guaranteed traffic, while introducing minimum delay and zero PDV (fig. 2).

Here, IHON is applied to a network segment of a C-RAN where FH traffic, i.e. CPRI flow encapsulated in Ethernet frames [7], is identified as the guaranteed service, with zero PDV, while BH traffic is dealt with preemption. IHON eliminates PDV of the FH traffic because the fixed delay $\Delta$ enables a time-window which gives sufficient time for processing and decision of $\mathrm{BH}$ packet preemption. This goes beyond, e.g., the IEEE 802.1Qbu preemption recommended in the IEEE 802.1CM standard for $\mathrm{FH}$, where $\mathrm{FH}$ packets may experience anyway PDV corresponding to the service time of 155 Byte. Hence, IHON is here characterized in relation to the C-RAN context with CPRI over Ethernet (CPRIoE). In particular, with reference to fig. 2, the $\mathrm{FH}$ inter-packet gap $T_{g a p}$ will be expressed in relation to CPRI characteristics.

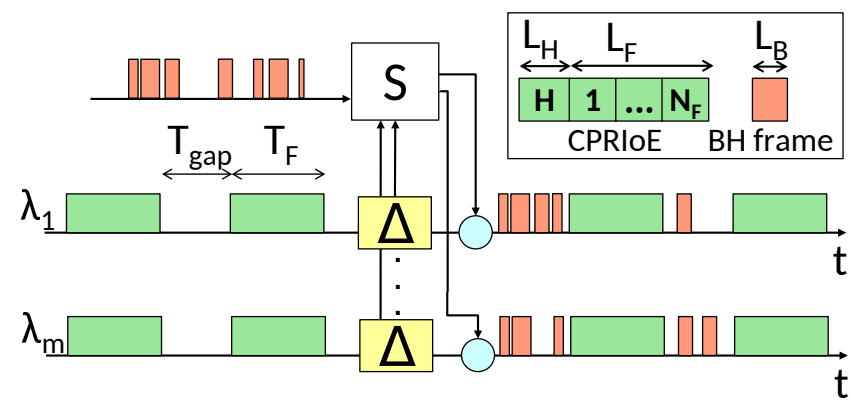

Fig. 2. IHON multiplexing scheme

\section{C-RAN TRAFFIC CHARACTERIZATION USING IHON}

CPRIoE traffic characterization has been analyzed in [7]. Parameters used in this study are reported in Tab.I. RRHs generate CPRI flows at different rates $\left(R_{C P R I}\right)$ set by the standard [2]. Each flow is composed of CPRI basic frames with fixed duration $T_{C P R I}=260 \mathrm{~ns}$. A certain number of CPRI basic frames $\left(N_{F}\right)$ are encapsulated in an Ethernet frame forming the CPRIoE payload of length: $L_{F}=N_{F} * R_{C P R I} *$ $T_{C P R I}$. CPRIoE frames are then sent by RRHs towards IHON switches, where they are delayed by $\Delta$. Also conventional $\mathrm{BH}$ traffic reaches the switches, loading the output channels with parameter $\rho_{B}$. The length of $\mathrm{BH}$ frames is considered to be exponentially distributed with parameter $L_{B}$. IHON switches

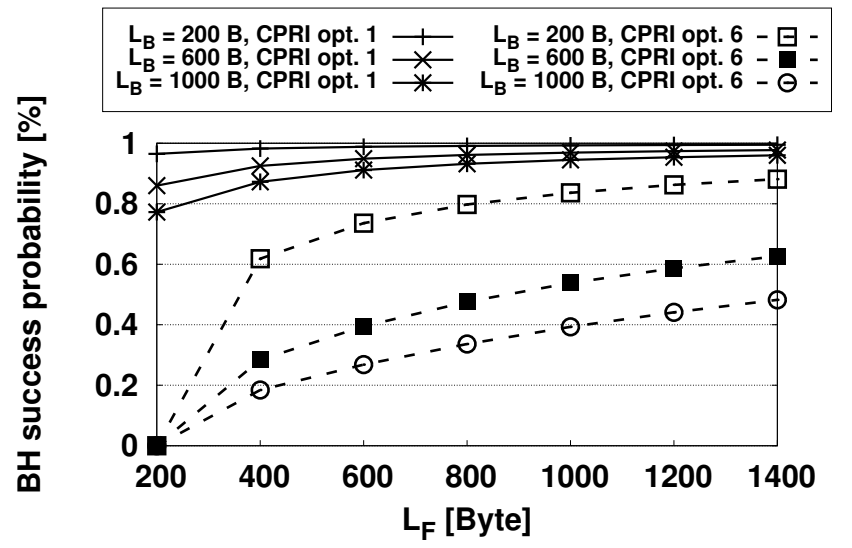

Fig. 3. BH success probability as a function of payload length $L_{F}$ for different BH payloads $L_{B}$ using CPRI opt. 1 and 6.

have $m$ output channels, each characterized by a rate $R_{W}$, and accommodates CPRIoE frames of duration: $T_{F}=\frac{H_{C P R I}+L_{F}}{R_{W}}$, where $H_{C P R I}$ is the header of CPRIoE frames assumed to be 44 Byte [7]. Depending on $N_{F}$ and $R_{C P R I}$, the gap duration $T_{\text {gap }}$ is selected according to: $T_{g a p}=\frac{L_{F}}{R_{C P R I}}-T_{F}$.

\section{Numerical Results}

To evaluate the benefits introduced by IHON, an eventdriven simulator has been developed. One RRH generates a CPRI flow according to two different options with rates $R_{C P R I}=614.4 \mathrm{Mbps}$ (option 1) and 6.144 Gbps (option 6). The IHON fixed delay $\Delta=99.2 n s$ is assumed, when not differently indicated, which corresponds to the smallest fragment (124 Byte) that can be preempted [9]. A single output channel $(m=1)$ with rate $R_{W}=10 \mathrm{Gbps}$ is considered. The number of CPRI basic frames in a guaranteed burst $N_{F}$ is varied over the intervals $[1,70]$ and $[1,7]$, for CPRI option 1 and 6, respectively [7], so that the payload length $L_{F}$ varies accordingly. A set of simulations varying the average BH packet length $L_{B}$ is obtained with a load $\rho_{B}$ such that a $\mathrm{BH}$ packet is always ready for transmission on the output channel.

Fig. 3 shows the success probability of the BH traffic, defined as the ratio between the packets not interrupted and the

TABLE I

IHON PARAMETERS.

\begin{tabular}{ll}
\hline \hline Parameter & Description \\
\hline$N_{F}$ & Number of CPRI basic frames forming a \\
$L_{F}$ & CPRIoE payload \\
$R_{W}$ & Payload length for CPRIoE frame \\
$T_{F}$ & Output channel rate \\
$T_{g a p}$ & CPRIoE duration \\
$\Delta$ & Gap duration \\
$\rho_{B}$ & Fixed delay to avoid collision \\
$L_{B}$ & Offered BH load per channel \\
$m$ & Average length of BH frames \\
\hline \hline
\end{tabular}




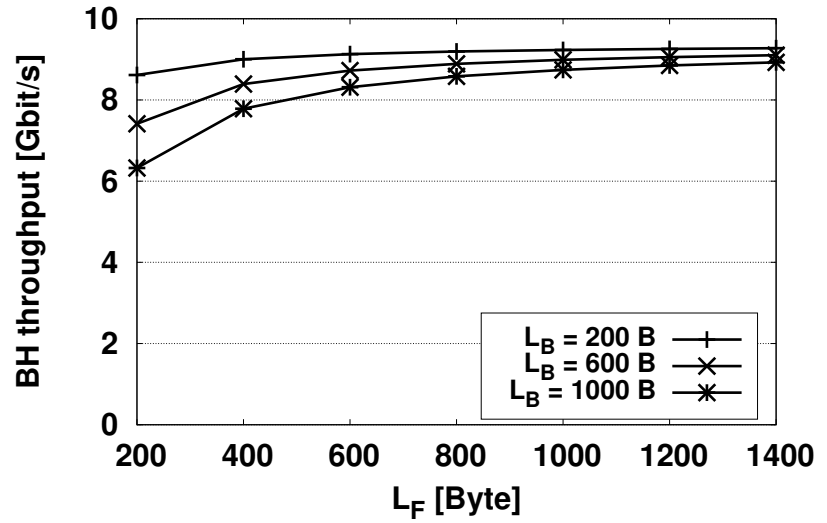

Fig. 4. BH throughput as a function of payload length $L_{F}$ for different $\mathrm{BH}$ payloads $L_{B}$ using CPRI opt. 1 .

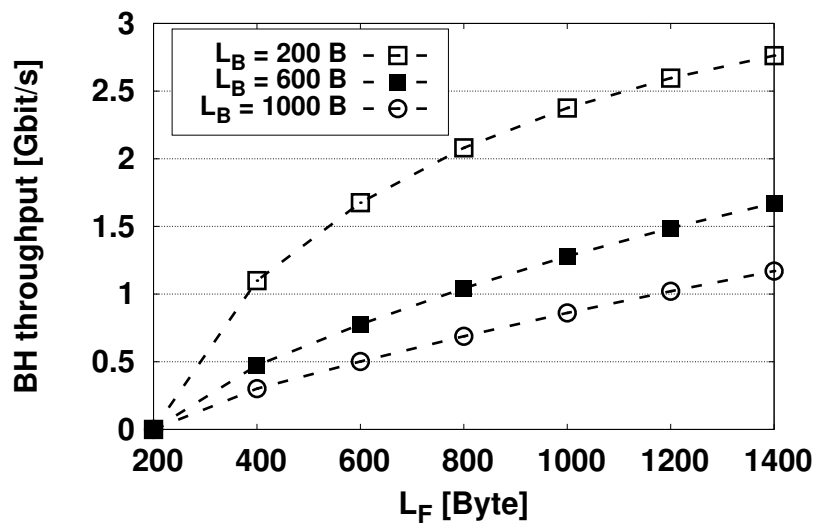

Fig. 5. BH throughput as a function of payload length $L_{F}$ for different $\mathrm{BH}$ payloads $L_{B}$ using CPRI opt. 6 .

total packets in service, as a function of $L_{F}$, for both CPRI options, varying $L_{B}$. In both cases, the success probability increases with $L_{F}$, due to the resulting larger $T_{g a p}$. Option 6 shows lower performance than option 1 due to the smaller size of the gap, especially when $L_{F}$ is low, so suggesting to use larger $N_{F}$ in this case. However, increasing $N_{F}$ increases the encapsulation delay, that may impact the maximum reach of FH connection. Figs. 4 and 5 report the BH throughput as a function of $L_{F}$ for option 1 and 6 respectively, varying $L_{B}$. In both cases, the throughput follows the trend of the success probability. In the case of option 1 the throughput reaches $9 \mathrm{Gbps}$ with quite limited influence of $L_{F}$ and $L_{B}$. With option 6 the influence of $\mathrm{BH}$ packet length increases. In particular, with large BH packets (e.g., $L_{B}=1000$ Byte) and small gaps (e.g., $L_{F}=200$ Byte) the exploitation of the left output channel capacity $\left(R_{W}-R_{C P R I}\right)$ by $\mathrm{BH}$ traffic is quite low. Fig. 6 shows the impact of $\Delta$ on $\mathrm{BH}$ throughput for CPRI option 6 when $L_{B}=600$ Byte. When $\Delta$ increases the $\mathrm{BH}$ throughput decreases, due to the shorter time available for $\mathrm{BH}$ traffic, which is switched off when a CPRIoE frame

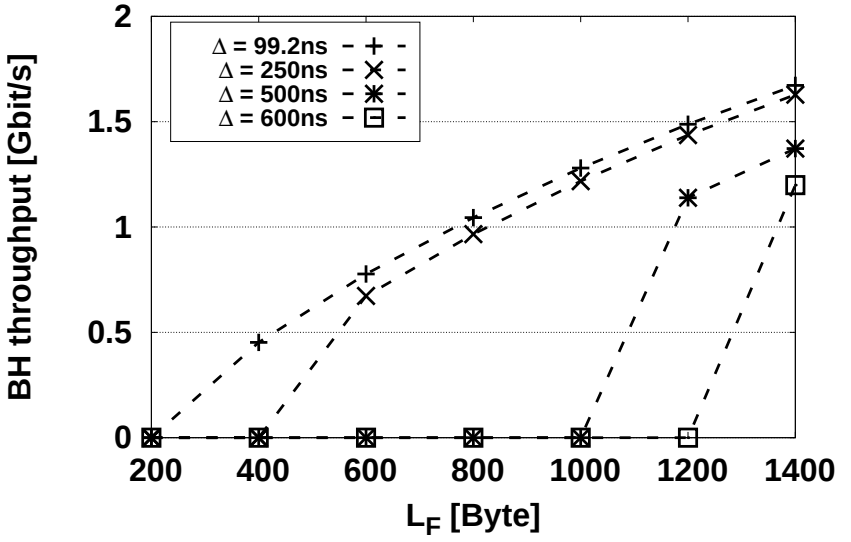

Fig. 6. BH throughput as a function of payload length $L_{F}$ for CPRI opt. 6 and different $\Delta$ when $\mathrm{BH}$ payloads $L_{B}=600 \mathrm{~B}$.

is detected and is waiting for transmission during the interval $\Delta$.

\section{Conclusions}

This paper has explored the feasibility of IHON extended with preemption in C-RAN for transporting $\mathrm{FH}$ traffic with timing transparency combined with preemptable $\mathrm{BH}$ traffic within the same optical Ethernet channel. Performance evaluations have been presented for different CPRI options, finding the amount of $\mathrm{BH}$ traffic taking advantage of unused $\mathrm{FH}$ capacity. Remarkable BH throughput is shown especially for CPRI option 1. Scheduling BH packets only when gaps in the $\mathrm{FH}$ traffic of suitable size for the $\mathrm{BH}$ packets are present, is an IHON characteristic. CPRI option 6 limits BH throughput because the smaller packet gaps in the FH traffic makes fitting of the $\mathrm{BH}$ traffic more difficult. Further investigations will include the introduction of controlled traffic mechanisms for adapting the $\mathrm{BH}$ traffic for better fitting the unused bandwidth in a FH multi-channel configuration.

\section{REFERENCES}

[1] A. Checko, et al., 'Cloud RAN for Mobile Networks. A Technology Overview',IEEE Communications Surveys and Tutorials, 17(1), 2015

[2] CPRI \& eCPRI, http://www.cpri.info/press.html, Jan. 2018.

[3] 3GPP TSG RAN WG3, TR 38.801, Apr. 2017.

[4] S. Bjornstad, et al., 'A packet switched hybrid optical network with service guarantees', IEEE JSAC, v.24, n.8, 2006.

[5] W. Cerroni, et al., 'Analytical model of quality of service scheduling for optical aggregation in data centers', Springer PNC, v.27, n.3, June 2014.

[6] R. Veisllari, et al., 'Field-trial demonstration of cost efficient subwavelength service through integrated packet/circuit hybrid network', IEEE/OSA JOCN, v.7, n.3, 2015.

[7] D. Chitimalla, et al., '5G fronthaul-latency and jitter studies of CPRI over ethernet', IEEE/OSA JOCN, v.9, n.2, 2017.

[8] R. Veisllari, et al., 'Experimental Demonstration of $100 \mathrm{~Gb} / \mathrm{s}$ Optical Packet Network for Mobile Fronthaul with Load-independent Ultra-low Latency', ECOC 2017.

[9] IEEE Standard P802.1CM, "Time Sensitive Networking for Fronthaul". 